\title{
Plantas com potencial medicinal e tóxico em comunidade atendida pelo Ambulatório Veterinário-UFPel*
}

\section{Potentially toxic and medicinal plants from community assisted in UFPel Veterinary Clinic}

\author{
Claudia Giordani, ${ }^{* *}$ Caroline Bohnen de Matos, ${ }^{* *}$ Karina Affeldt Guterres, ${ }^{* *}$ Cristine Cioato da Silva, ${ }^{* *}$ Rosema Santin, ${ }^{* *}$ \\ Luiz Filipe Damé Schuch, ${ }^{* *}$ Marlete Brum Cleff**
}

\begin{abstract}
Resumo
O objetivo deste trabalho foi identificar plantas utilizadas como medicinais e aquelas com potencial tóxico localizadas no bairro Simões Lopes, Padre Réus e Porto em Pelotas-RS. Durante um ano foram entrevistados 111 proprietários de animais atendidos no Ambulatório Veterinário-Universidade Federal de Pelotas, com o propósito de avaliar o conhecimento, cultivo, utilização e forma de obtenção das plantas medicinais, faixa etária e o sexo das pessoas, número de animais nas residências e resultados do uso de plantas nos animais. Após investigação inicial, realizaram-se visitas na comunidade com intuito de fotografar e coletar amostras das plantas para identificação botânica. Foram listadas 74 plantas com fins medicinais, principalmente das famílias Asteraceae e Lamiaceae, usadas em minoria nos animais. As principais indicações de uso foram para o sistema digestório e tegumentar, seguido do musculoesquelético e respiratório, sob forma de infusão e decocto. Grande parte dos entrevistados desconhecia os efeitos adversos do uso inadequado de plantas medicinais, e espécies diferentes eram conhecidas com o mesmo nome popular, podendo resultar em toxicidade, dosagens errôneas e ações desconhecidas. Em relação ao cultivo e utilização das plantas medicinais, predominava em pessoas com idade média de 41,2 anos, que obtiveram o conhecimento principalmente de pais e avós. As plantas com potencial tóxico encontradas pertenciam às famílias Araceae, Euphorbiaceae e Araliaceae, totalizando 20 espécies; porém, apenas seis foram citadas como tóxicas pelos entrevistados. Assim, observou-se a existência e uso de muitas plantas medicinais, além da presença de espécies potencialmente tóxicas na região, sendo imprescindível ressaltar, com embasamento científico, possíveis benefícios e riscos da administração em animais.
\end{abstract}

Palavras-chave: animais, fitoterapia, intoxicação.

\begin{abstract}
The aim of this study was to identify plants used in traditional medicine, as well as those with toxic potential in the neighborhoods of Simões Lopes, Padre Réus and Porto in the city of Pelotas. For a year there were interviewed 111 owners of animals treated at the Veterinary Clinic-Universidade Federal de Pelotas, in order to assess the knowledge, cultivation, use and form of obtaining medicinal plants, age and sex of people, number of animals and results of the use of plants in the animals. After initial investigation, visits were held in the community aiming to shoot and collect samples of plants for botanical identification. They were listed 74 plants for medicinal purposes, mainly from Asteraceae and Lamiaceae families, minimally used in animals. The main indications of use were for digestive and integumentary system, followed by musculoskeletal and respiratory, by infusion and decoction. Most of the interviewers were unaware of the adverse effects of inappropriate use of medicinal plants, and different species were known with the same common name, so they could result in toxicity, wrong dosages and unknown actions. There was a predominance of cultivation and use of medicinal plants in people with an average age of 41.2 years who obtained knowledge mainly from parents and grandparents. The toxic potential plants found were from Araceae, Euphorbiaceae and Araliaceae families, in a total of 20 species, but only six were cited as toxic by the interviewees. There was noticed the existence and use of many medicinal plants and presence of potentially toxic species in the region; so it is essential to evidence scientifically possible benefits and risks of administration in animals.
\end{abstract}

Keywords: animals, phytotherapy, intoxication.

\section{Introdução}

Historicamente, o uso de plantas acompanha a evolução humana, tanto para a alimentação, como construção de moradias, confecção de vestimentas, e principalmente no tratamento de enfermidades em pessoas e animais (Balick e
Cox, 1997; Cleff, 2008; Lopes et al., 2010). Atualmente, com a sobre-exploração dos recursos, introdução de espécies exóticas, crescimento populacional, registra-se a perda de habitat de espécies vegetais nativas (Ganem, 2010). Neste cenário, o resgate, manutenção e organização do saber popular acerca das plantas assumem papel indispensável (Borsato et al., 2009).

\footnotetext{
${ }^{*}$ Recebido em 18 de maio de 2015 e aceito em 8 de fevereiro de 2017.

**Universidade Federal de Pelotas, Faculdade de Veterinária, Caixa Postal 354 - Campus Universitário S/Nº. CEP 96 010-900 Capão do Leão/RS.

Autor para correspondência: claarte@hotmail.com
} 
De acordo com a Organização Mundial de Saúde, as plantas medicinais deveriam ser a melhor fonte para obter fármacos (Prashar et al., 2003), sendo nos últimos anos implementados políticas e programas que visam tanto o treinamento para cultivo, coleta, processamento, armazenamento e manipulação de plantas medicinais, como também sua aplicação na atenção primária à saúde, tendo como objetivo utilizá-las como auxiliar no tratamento de enfermidades (Matos, 1998; Batista e Valença, 2012). No Brasil, a Agência Nacional de Vigilância Sanitária tem regulamentado o uso de algumas plantas medicinais após estudos científicos comprobatórios de eficácia em determinadas enfermidades e ou sinais clínicos (Brasil, 2011). Além disso, a busca de novas opções terapêuticas frente a micro-organismos resistentes, associado ao alto custo dos medicamentos, estimula as pesquisas acerca do uso das plantas medicinais (Albuquerque e Hanazaki, 2006; Oliveira et al., 2009; Zago et al., 2009). Entretanto, muitas plantas são consumidas pela população ou oferecidas aos animais sem nenhuma orientação, expondo-os a riscos, visto que várias espécies são potencialmente tóxicas (Arnous et al., 2005; Schwamback e Amador, 2007). No Brasil existe em torno de 113 descrições de plantas tóxicas, e as plantas ornamentais são aquelas mais relatadas em intoxicações de pequenos animais (Riet-Correa et al., 2007; Riboldi, 2010).

Assim, o objetivo deste trabalho foi identificar plantas utilizadas para fins medicinais e aquelas com potencial tóxico encontradas na região de abrangência do Ambulatório Veterinário da Universidade Federal de Pelotas (UFPel).

\section{Material e métodos}

O trabalho foi desenvolvido no período de 2011 a 2012 em Pelotas, Rio Grande do Sul, Brasil, através de entrevistas realizadas com tutores de animais atendidos pelo Ambulatório VeterinárioUFPel. O Ambulatório está localizado em uma comunidade considerada em vulnerabilidade socioeconômica inserida na periferia do centro da cidade (31 $46^{\circ} 39.3^{\prime}$ S e $52^{\circ} 21^{\prime} 03.4^{\prime \prime} \mathrm{W}$ ) abrangendo os bairros Simões Lopes, Padre Réus e Porto. O Ambulatório está vinculado ao Hospital de Clínicas Veterinária da UFPel, com média de 60 atendimentos mensais. Para ser inserido no atendimento ambulatorial, era necessário que o(s) proprietário(s) do(s) animal(is) comprovasse(ssem) idade igual ou superior a 18 anos, renda inferior a um salário mínimo e residência na região em torno do Ambulatório. $O$ atendimento compreendia o turno da manhã, dois dias por semana, período no qual os profissionais realizavam consultas clínicas aos animais, além de orientação aos proprietários.

Os tutores de animais atendidos no Ambulatório Veterinário foram convidados a participar da pesquisa, e após aceitação, através da assinatura do Termo de Consentimento Livre e Esclarecido (TCLE), iniciava-se a entrevista. O questionário aberto continha as seguintes perguntas: Qual sua idade?
Sexo feminino ou masculino? Possui quantos animais na residência? Quais espécies? Possui conhecimento sobre plantas medicinais? Usa e cultiva plantas? Quais? Forma de aquisição? Utilização no tratamento de pessoas e animais? De que forma? Para quais distúrbios ou enfermidades? Resultados obtidos após o uso das plantas? A aplicação do questionário foi realizada por professores e estudantes envolvidos no projeto.

Após este período de investigação, também com o consentimento dos moradores através do TCLE, foram realizadas visitas na comunidade, juntamente com a assistente social da UFPel, para fotografar e coletar amostras das plantas. As amostras foram encaminhadas ao Departamento de Botânica do Instituto de Biologia-UFPel para identificação das espécies.

\section{Resultados e discussão}

Durante o estudo, havia 500 pessoas cadastradas no projeto de atendimento, sendo entrevistados 111 proprietários de animais, $65(58,6 \%)$ mulheres e $46(41,4 \%)$ homens, com idade entre 18 e 75 anos (média de 36,27 anos), dos quais possuíam um total de 322 animais de companhia e/ou de produção, correspondendo a uma média de 2,9 animais por residência, entre eles cães, gatos, cavalos, bovinos, ovinos e aves, sendo os cães e cavalos as espécies mais numerosas ( $90 \%$ e $60 \%$, respectivamente). Demonstrando assim, um bom potencial para a aplicação de plantas medicinais no tratamento de enfermidades dos animais domésticos, já que o custo dos medicamentos muitas vezes inviabilizava o tratamento.

Em relação às plantas medicinais, foram listadas 74 plantas pelo seu nome popular, principalmente das famílias Asteraceae e Lamiaceae, conhecidas ou utilizadas como medicinais pela população do estudo, sendo estas as mais cultivadas conforme Severiano et al. (2010). As plantas foram citadas tanto para uso próprio como também para os animais, sob diferentes formas e finalidades (Tabela 1). A comunidade faz uso de várias espécies vegetais $(n=74)$, o que também foi observado por Santos et al. (2008), ao realizar um levantamento de espécies medicinais em uma comunidade rural, onde foi relatada a utilização de 115 plantas. Noventa por cento dos entrevistados desconheciam os efeitos tóxicos relacionados com o uso inadequado e a superdosagem das plantas medicinais e também a possibilidade de interação medicamentosa destas com fármacos alopáticos. O volume de chá administrado aos animais era, na maioria das vezes, a mesma quantidade que os proprietários utilizavam para si mesmos, ou seja, uma xícara/dose. Isso determina um ponto crítico na terapia medicinal popular, podendo expor os usuários a quadros de hipersensibilidade, efeitos tóxicos e até o óbito (Veiga Júnior et al., 2005), sendo este fato preocupante, já que na comunidade estudada muitas vezes os recursos naturais eram citados como única opção viável para tratamento das enfermidades de pessoas e animais, devido à baixa renda familiar. 
Tabela 1: Plantas conhecidas como medicinais, parte utilizada e indicações terapêuticas citadas pelos proprietários de animais atendidos no Ambulatório Veterinário - UFPel

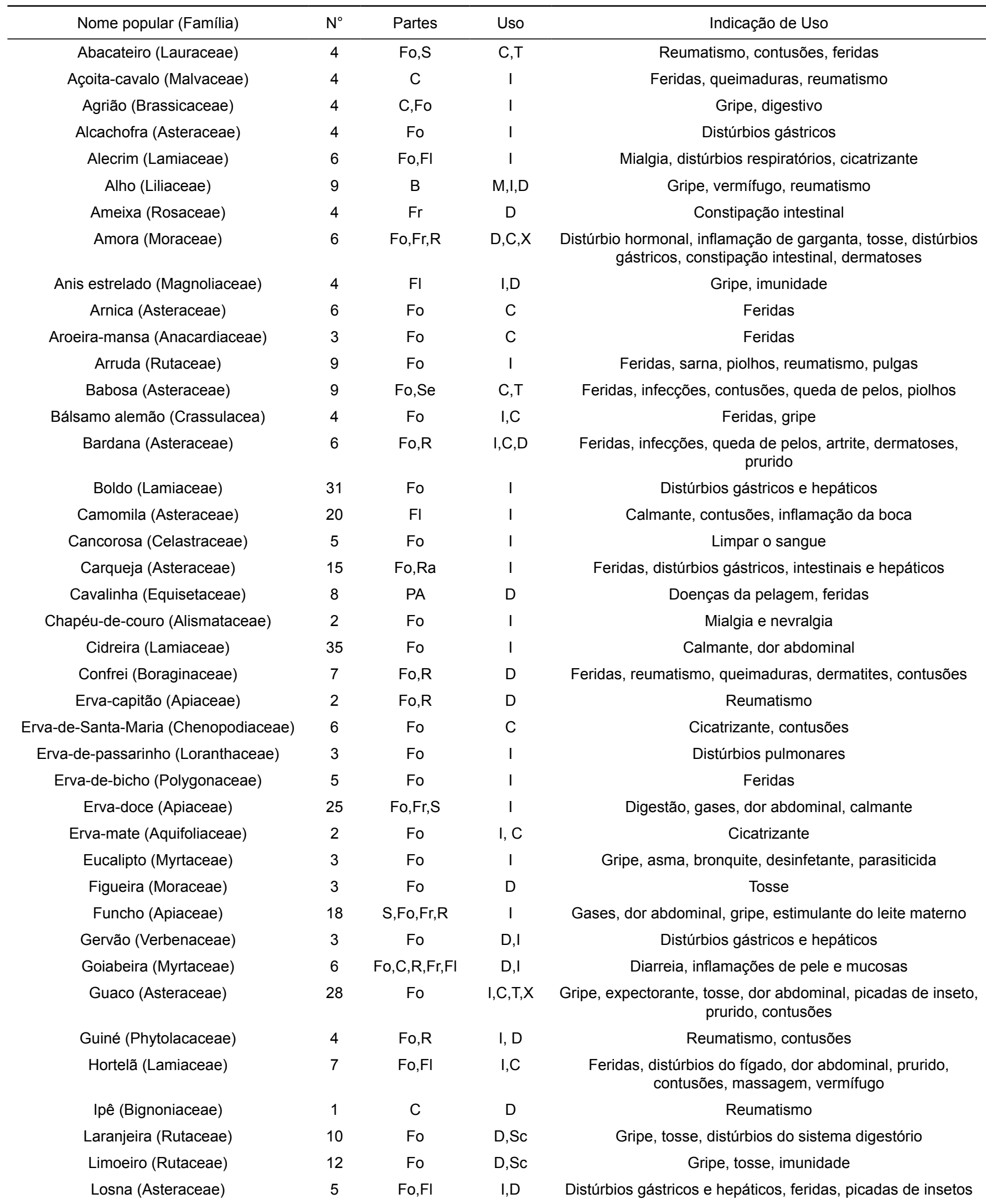




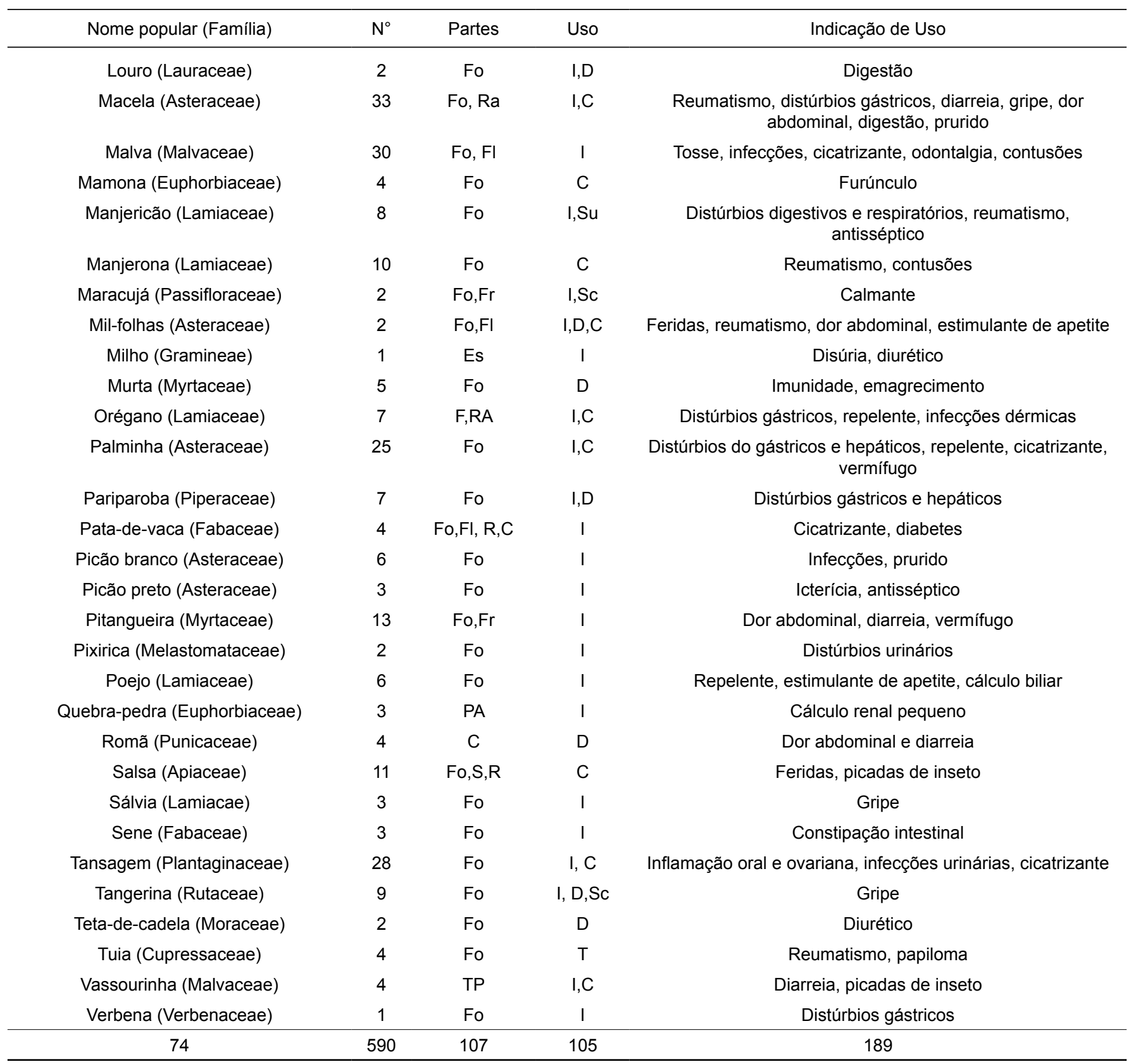

${ }^{*} N^{\circ}$ : número de citações; B: Bulbo; C: Casca; Es: Estilo-estigma; Fl: Flores; Fo: Folhas; Fr: Frutos; R: Raiz; Ra: Ramos; S: Semente; Se: Seiva; PA: Partes aéreas; TP: Toda planta; I: infusão; D: decocção; C: cataplasma e compressas; Sc: suco; Su: sumo; T: tintura; M: maceração, X: xarope.

As plantas medicinais mais citadas pela população foram macela $(44,5 \%)$, boldo $(41,8 \%)$, malva $(40,5 \%)$, guaco $(34,8 \%)$ e tansagem (34,8\%). Quanto à parte vegetal mais utilizada, destacou-se o uso das folhas por $82,1 \%(n=78)$ dos entrevistados, concordando com levantamento feito por Sevignani e Jacomassi (2003), resultado da facilidade e disponibilidade de coleta, sendo também uma boa prática em relação à preservação das espécies (Castellucci et al., 2000; Jacoby et al., 2002).

Os entrevistados citaram que as plantas eram utilizadas principalmente para distúrbios gastrointestinais $(29,1 \%)$, dermatológicos
(28,0\%), seguido de distúrbios musculoesqueléticos $(15,8 \%)$ e respiratórios (13,7\%). As principais formas de preparação para o uso foram a infusão e decocto, corroborando com Vendruscolo e Mentz (2006), que observaram a utilização de ambas as formas pela maioria dos informantes.

Em relação aos resultados pós-uso das plantas como terapia única, foi citado melhora em $94 \%$ dos casos e $6 \%$ de reações adversas e ineficácia do tratamento. Tais dados divergem dos encontrados por Lima e colaboradores (2012), onde $100 \%$ dos entrevistados não apresentaram efeito colateral e relataram 
melhora dos sintomas após o uso, e destes, 67,95\% associavam o uso de plantas com a terapia alopática.

Considerando o grupo de pessoas entrevistadas (111/100\%), existia um grande grupo que utilizava plantas (95/85,5\%), e destes, menos da metade utilizava as plantas em animais (28/25,2\%), divergindo de Lima et al. (2012), que demonstrou que dos entrevistados em seu estudo, cerca de $48 \%$ usavam as plantas para tratar seus animais.

O pouco uso das plantas em animais pode ser explicado pela falta de incentivo e orientação por parte dos médicos veterinários, como descreve Almeida e Freitas (2006), que ao fazer uma análise do meio acadêmico do médico veterinário em formação, observou que o conhecimento sobre o uso de plantas medicinais para terapia é mínimo, e no que tange à prática clínica, este é extremamente reduzido.

Entretanto, aos poucos, o uso das plantas medicinais em veterinária tem ganhado espaço, principalmente através do incentivo e correlação do saber popular à pesquisa científica, com o propósito de gerar novas possibilidades na terapia das enfermidades dos animais (Marinho et al., 2007; Silva et al. 2013).

Quanto ao conhecimento do uso das plantas como forma terapêtica, os entrevistados informaram ter adquirido por intermédio da família (pais e avós) em $85 \%(n=81)$ dos casos, e por intermédio de revistas, reportagens e livros em $15 \%(n=14)$. Estas formas de obtenção do conhecimento também foram observadas em estudo anterior (Lopes et al., 2012), diferindo apenas quanto ao principal membro da família que transfere o conhecimento. Em nossos resultados, $70 \%$ dos casos foram adquiridos pelos pais e apenas $15 \%$ pelos avós, enquanto que no estudo de Lopes e colaboradores (2012) a informação foi transmitida principalmente pelos avós.

Com relação à distribuição das plantas, 78 das residências da população entrevistada cultivavam plantas, com média de 4,4 espécies vegetais, e destas que possuíam plantas no quintal, 77 faziam uso para tratamento. Além disso, as outras 33 moradias, que não cultivavam plantas, 17 adquiriam-nas em ervateiro, parentes, amigos, vizinhos, supermercado, farmácia e ou de áreas rurais.

O grande índice de pessoas com plantas medicinais em suas residências pode ser explicado pela miscigenação de culturas e também pelas condições do clima e solo no Rio Grande do Sul que favorecem o cultivo de diversas espécies vegetais, o que difere de regiões como o estado do Maranhão, onde levantamento realizado em bairros da cidade de São Luís demonstrou o baixo número de residências com plantas medicinais (Pessoa e Cartágenes, 2010). Ao mesmo tempo pode ser observado que pessoas que tinham plantas em casa, também adquiriam de outras fontes, o que demonstra o interesse e a confiança que as pessoas têm no uso das plantas medicinais, concordando com o observado por Quevedo e colaboradores (2011).

Mediante os dados da pesquisa, a faixa etária média dos entrevistados que cultivavam e utilizavam plantas medicinais era de 41,4 anos, e dos que não plantavam e não faziam uso destas, 24 anos. Isto ocorre pelo maior contato dos adultos com os idosos da comunidade, demonstrando também a falta de interesse dos mais jovens sobre a importância das plantas. As pessoas mais jovens, em sua grande maioria, não utilizavam as plantas, não tinham conhecimento ou não souberam informar a respeito. Além disso, pessoas que passaram por um período de suas vidas na zona rural, e que pertenciam ao sexo feminino, foram aquelas que possuíam maiores conhecimentos e usavam com frequência as plantas com fins terapêuticos, mantendo inclusive pequenos canteiros em suas residências.

Existe uma grande importância de estabelecer um resgate e preservação das espécies medicinais, principalmente pelo fato de serem fontes de pesquisa de novos compostos bioativos, abrindo a possibilidade de utilizá-las como alternativa terapêutica (Foglio et al., 2006; Hoeffel et al., 2011). Desta forma, é de suma importância a interação entre as comunidades, distribuindo amplamente esse conhecimento e incidindo diretamente na promoção da saúde humana e animal (Souza et al., 2012).

A visita à comunidade ocorreu nas residências onde os proprietários aceitaram participar do projeto $(n=39)$, sendo observadas plantas com potencial terapêutico $(n=40)$ e tóxico $(n=20)$, encontradas nos jardins ou proximidades das casas. Nesta etapa foram coletadas 32 amostras de plantas utilizadas como medicinais pela população e identificadas botanicamente, sendo elas: Achiella millefolium (Mil-folhas), Artemisia absinthium (Losna), Arctium minus (Bardana), Bauhinia forficata (Pata-devaca), Baccharis trimera (Carqueja), Bidens pilosa (Picão-preto), Coix lacryma-jobi (Lágrima-de-Nossa-Senhora), Cupressus sempervirens (Cipreste), Cymbopogon citratus (Capim-cidreira), Equisetum hyemale (Rabo-de-lagarto), Eugenia uniflora (Pitangueira), Hydrocotyle bonariensis (Erva-capitão), Juncus bufonius (Junco), Luehea divaricata (Açoita-cavalo), Majorana hortensis (Manjerona), Mentha pulegium (Poejo), Origanum vulgare (Orégano), Ocimum basilicum (Manjericão), Pfaffia glomerata (Ginseng), Piper umbellatum (Pariparoba), Plantago australis (Tansagem), Plectranthus ornatus (Boldo), Plectranthus barbatus (Boldo), Polygonum hydropiperoides (Erva-de-bicho), Psidium guajava (Goiabeira), Rosmarinus officinalis (Alecrim), Schinus terebinthifolius (Aroeira-mansa), Senna corymbosa (Fedegoso), Solidago chilensis (Lanceta), Thuja orientalis (Tuia), Tripogandra diuretica (Trapoeraba) e Verbena litoralis (Verbena). Algumas destas plantas, citadas como medicinais, tinham as formas de uso e indicações terapêuticas desconhecidas por parte dos entrevistados.

Das plantas analisadas botanicamente, duas (Picão-branco e Picão-preto) foram citadas por nomes populares diferentes, mas correspondiam à mesma espécie (Bidens pilosa), e outras duas plantas citadas como sendo a mesma (Boldo), correspondiam a espécies diferentes (Plectranthus ornatus e Plectranthus barbatus). Isso pode representar um risco à saúde, pelo consumo ou administração aos animais sem identificação botânica e orientação, expondo os consumidores à toxicidade, dosagens errôneas e ações desconhecidas (Arnous et al., 2005; Schwamback e Amador, 2007; Verdam e Silva, 2010), sendo necessário informar os proprietários, através de conhecimento científico, dos benefícios e riscos que as plantas podem causar.

Com relação às plantas com potencial tóxico, foram listadas apenas seis espécies pela população, sendo elas Aroeirabrava (Lithraea brasiliensis - Anacardiaceae), Cinamomo (Melia azedarach - Meliaceae), Comigo-ninguém-pode (Dieffenbachia 
picta - Araceae), Copo-de-leite (Zantedeschia aethiopica Araceae), Mamona (Ricinus communis - Euphorbiaceae) e Samambaia (Pteridium aquilinum - Polypodiaceae). Porém, nas visitações foram encontradas mais 14 espécies consideradas tóxicas: Antúrio (Anthurium andraeanum - Araceae), Azaléia (Rhododendron spp. - Ericaceae), bico-de-papagaio (Euphorbia pulcherrima - Euphorbiaceae), Cheflera (Schefflera spp. Araliaceae), Coroa-de-Cristo (Euphorbia milii - Euphorbiaceae), Espada-de-São-Jorge (Sansevieria trifasciata - Liliaceae), Hera (Hedera helix - Araliaceae), Hortência (Hydrangea macrophylla - Saxifragaceae), Kalanchoe (Kalanchoe spp. - Crassulaceae), Lantana (Lantana spp. - Verbenaceae), Lírio-da-paz (Spathiphyllum wallisii - Araceae), Trevo-branco (Trifolium repens - Fabaceae), Trombeteira (Datura suaveolens - Solanaceae) e Unha-de-gato (Ficus pumila - Moraceae), totalizando 20 espécies tóxicas.

Mediante esses dados, percebe-se que com as entrevistas e visitações às moradias, cerca de $80 \%(n=89)$ das pessoas têm pelo menos uma espécie tóxica em sua residência ou proximidades, e ainda, $70 \%(n=14)$ das plantas não foram citadas como tóxicas, confirmando o desconhecimento sobre o risco que estas representam, conforme descrito por Vasconcelos et al. (2009). A presença destas plantas torna imprescindível o conhecimento de seu caráter tóxico, pois de acordo com Riboldi (2010), grande porcentagem destas plantas estão envolvidas em acidentes tóxicos em cães e gatos.

\section{Agradecimentos}

À Prof ${ }^{a}$ Raquel Lüdtke, PPGV-UFPel, CAPES, CNPq e FAPERGS.

\section{Referências}

ALBUQUERQUE U.P.; HANAZAKI, N. As pesquisas etnodirigidas na descoberta de novos fármacos de interesse médico $e$ farmacêutico: fragilidades e perspectivas. Revista Brasileira de Farmacognosia, v. 16, p. 678-689, 2006.

ALMEIDA, K.S.; FREITAS, F.L.C. Etnoveterinária: a fitoterapia na visão do futuro profissional veterinário. Revista Verde de Agroecologia e Desenvolvimento Sustentável, v.1, n.1, p.67-74, 2006.

ARNOUS, A.H.; SANTOS, A.S.; BEINNER, R.P.C. Plantas Medicinais de Uso Caseiro - Conhecimento Popular e Interesse por Cultivo Comunitário. Revista Espaço para a Saúde, Londrina, v. 6, n. 2, p. 1-6, 2005.

BALICK, M.J.; COX, P.A. Plants, people and culture. New York: Scientific American Library. 1997. 228 p.

BATISTA, L.M.; VALENÇA, A.M.G. A Fitoterapia no Âmbito da Atenção Básica no SUS: Realidades e Perspectivas. Pesquisa brasileira em Odontopediatria e Clínica integrada, v. 12, n. 2, p. 293-296, 2012.

BORSATO, A.V.; SILVA, A.; SANTOS, A.G.; JORGE, M.H.A. Plantas Medicinais e Agroecologia: Uma Forma de Cultivar o Saber Popular na Região de Corumbá, MS. Corumbá: Embrapa Pantanal, Documentos, n.103, 2009. 12 p.

BRASIL, Agência Nacional de Vigilância Sanitária. Formulário de Fitoterápicos da Farmacopéia Brasileira / Agência Nacional de Vigilância Sanitária. Brasília: ANVISA, 2011. 126 p.
Outra questão importante a ser salientada é a dificuldade do médico-veterinário em estabelecer uma terapia específica e direcionada para a intoxicação, pois os animais inicialmente apresentam sinais clínicos variados, e devido ao desconhecimento do proprietário, este não relaciona estes sinais com a ingestão ou contato com as plantas. Segundo a literatura, os sinais são confundíveis com outras doenças ou envenenamentos, como vômito, salivação, diarreia, letargia e até arritmias (Milewski e Khan, 2006; Martins et al. 2013). De acordo com os dados dos atendimentos do Ambulatório Veterinário-UFPel, algumas das plantas encontradas na comunidade, já estiveram envolvidas em diagnósticos de intoxicação em cães e gatos, como o cinamomo, comigo-ninguém-pode e lírio-da-paz. Estando o presente estudo, de acordo com a maior incidência de intoxicações com a planta comigo-ninguém-pode, relatada pelo Centro de Informações Toxicológicas do Rio Grande do Sul (CIT/RS, 2013).

\section{Conclusões}

Na região de abrangência do Ambulatório Veterinário da Universidade Federal de Pelotas observa-se que a população conhece e utiliza muitas espécies de plantas como medicinais, além de estarem presentes várias plantas potencialmente tóxicas, destacando-se a importância de educação, com base científica, acerca dos possíveis benefícios e riscos quanto ao uso destas nos animais.
CASTELLUCCI, S.; LIMA, M.I.S.; NORDI, N.; MARQUES, J.G.W. Plantas medicinais relatadas pela comunidade residente na Estação Ecológica de Jataí, município de Luís Antonio - SP; uma abordagem etno-botânica. Revista Brasileira de Plantas Medicinais, v. 3, n. 1, p. 51-60. 2000.

Centro de Informações Toxicológicas do Rio Grande do Sul (CIT/ RS). NICOLELLA, A.; FERREIRA, E.M.; LESSA, C.A.S. Relatório Anual 2013 - Dados de Atendimento. Centro de Informações Toxicológicas do Rio Grande do Sul (CIT/RS). Disponível em: <http://www.cit.rs.gov.br/>. Acessado em: 10 de jan. 2015.

CLEFF, M.B. Avaliação da atividade antifúngica do óleo essencial de Origanum vulgare $L$. frente a fungos de importância em veterinária com ênfase emCandidaspp. 2008. 129 f. Tese (Doutorado em Ciências Veterinárias) - Faculdade de Veterinária Universidade Federal do Rio Grande do Sul, Porto Alegre, 2008.

FOGLIO, M.A.; QUEIROGA, C.L.; SOUZA, I.M.O; RODRIGUES, R.A.F. Plantas medicinais como fonte de recursos terapêuticos: Um modelo multidisciplinar. Multiciência, n. 7, 2006.

GANEM, R.S. Conservação da biodiversidade: legislação e políticas públicas. Brasília: Câmara dos Deputados, Edições Câmara. 2010. 437 p.

HOEFFEL, J.L.M.; GONÇALVES, N.M.; FADINI, A.A.B.; SEIXAS, S.R.C. Conhecimento tradicional e uso de plantas medicinais na APA'S Cantareira/SP e Fernão Dias/MG. Revista VITAS - Visões Transdisciplinares sobre Ambiente e Sociedade, n.1, setembro, 2011. 
JACOBY, C.; COLTRO, E.M.; SLOMA, D.C.; MULLER, J.; DIAS, L.A.; LUFT, M.; BERUSKI, P.; NETO, R.M.R. Plantas medicinais utilizadas pela comunidade rural de Guamirim, Município de Irati, PR. Revista Ciências Exatas e Naturais. v. 4, n.1, p.1-7. 2002.

LIMA, R.P.; PALITOT, K.M.; REGO, M.A.E.; XAVIER, F.J.R.; SOUZA, A.E.F. Emprego de plantas medicinais em animais de companhia e de produção da zona rural do município de Juru-PB. Revista de Biologia e Farmácia, v.8, n. 1, p. 85-92, 2012.

LOPES, G.A.D.; FELICIANO, L.M.; DINIZ, R.E.S.; ALVES, M.J.Q.F. Plantas Medicinais: Indicação Popular de Uso no Tratamento de Hipertensão Arterial Sistêmica (HAS). Revista Ciência em Extensão, v. 6, n. 2, p. 143, 2010.

LOPES, I.S.; SILVA, J.E.R.; MACHADO, I.A.; SILVA, C.E.M.R.; MARINHO, M.G.V.; RANGEL, J.A.F. Levantamento de plantas medicinais utilizadas na cidade de Itapetim, Pernambuco, Brasil. Revista de Biologia e Farmácia, v. 7, n. 1, p. 115-121, 2012.

MARINHO, M.L.; ALVES, M.S.; RODRIGUES, M.L.C.; ROTONDANO, T.E.F.; VIDAL, I.F.; SILVA, W.W.; ATHAYDE, A.C.R. A utilização de plantas medicinais em medicina veterinária: um resgate do saber popular. Revista Brasileira de Plantas Medicinais, v. 9, n. 3, p. 64-69, 2007.

MARTINS, D. B.; MARTINUZZI, P. A.; SAMPAIO, A. B.; VIANA, A. N. Plantas tóxicas: uma visão dos proprietários de pequenos animais. Arquivos de Ciências Veterinárias e Zoologia da UNIPAR, v. 16, n. 1, p. 11-17, 2013.

MATOS, F.J.A. Farmácias vivas: sistema de utilização de plantas medicinais projetado para pequenas comunidades. 3. ed. Fortaleza: EUFC, 1998. 219 p.

MILEWSKI, L.M.; KHAN, S.A. An overviewof potentially lifethreatening poisonous plants in dogs and cats. Journal of Veterinary Emergency and Critical Care, v.16, n.1, p. 25-33, 2006.

OLIVEIRA, F.C.F.; ALBUQUERQUE, U.P.; FONSECA-KRUEL, V.S.; HANAZAKI, N. Avanços nas pesquisas etnobotânicas no Brasil. Acta Botanica Brasilica, v. 23, n. 2, p. 590-605, 2009.

QUEVEDO, M.D.; GONÇALVES, R.F.; GONZALES, F. O conhecimento sobre a utilização de plantas medicinais em dois municípios do litoral de São Paulo, SP. Revista Ceciliana, v. 3, n.2, p. 35-39, 2011.

PESSOA, D.L.R.; CARTÁGENES, M.S.S. Utilização de plantas medicinais por moradores de dois bairros na cidade de São Luís, estado do Maranhão. Enciclopédia Biosfera, Centro Científico Conhecer, v. 6, n. 11, p. 1-9, 2010.

PRASHAR, A.; HILI, P.; VENESS, R.G.; EVANS, C.S. Antimicrobial action of palmarosaoil (Cymbopogon martinii) on Saccharomyces cerevisiae. Phytochemistry, v. 63, n. 5, p. 569575, 2003.

RIBOLDI, E.O. Intoxicações em pequenos animais: uma revisão. 2010. 118f. Monografia (Graduação) - Faculdade de Veterinária, Universidade Federal do Rio Grande do Sul, Porto Alegre, 2010.
RIET-CORREA, F.; MEDEIROS, R.M.T.; TOKARNIA, C.H.; DÖBEREINER, J. 2007. Toxic plants for livestock in Brazil: economic impact, toxic species, control measures and public health implications. In: Panter KE, Wierenga TL, Pfister JA. (Eds). Poisonous Plants: global research and solutions. Wallingford: CAB International, 2007. p. 2-14.

SANTOS, J.F.L.; AMOROSO, M.C.M.; MING, L.C. Uso popular de plantas medicinais na comunidade rural de Bargem Grande, Município de Natividade da Serra, SP. Revista Brasileira de Plantas Medicinais, v. 10, n. 3, p. 67-81, 2008.

SCHWAMBACK, K.H.; AMADOR, T.A. Estudo da utilização de plantas medicinais e medicamentos em um município do sul do Brasil. Latin American Journal Of Pharmacy, v. 26, n. 4, 602-608, 2007.

SEVERIANO, M.V.N.; DANTAS, I.C.; SILVA, J.C.; FELISMINO, D.C. Levantamento das plantas medicinais cultivadas no Centro de Estudo e Pesquisa Malaquias da Silva Amorim. Revista Brasileira de Biologia e Farmácia, v. 4, n. 1, p. 93-101, 2011.

SEVIGNANI, A.; JACOMASSI, E. Levantamento de plantas medicinais e suas aplicações na vila rural "Serra dos Dourados" Umuarama/PR. Arquivos de Ciências da Saúde da UNIPAR, v. 7, n. 1, p. 27-31, 2003.

SILVA, W.M.O.; SOUZA, G.F.X.T.; VIEIRA, P.B.; SANAVRIA, A. Uso popular de plantas medicinais na promoção da saúde animal em assentamentos rurais de Seropédica-RJ. Revista Brasileira de Ciência Veterinária, v. 20, n. 1, p. 32-36, 2013.

SOUZA, A.E.F.; NASCIMENTO, H.H.L.; MAYER, K.D.G.; GOMES, M.K.O. Etnobotânica: Importância do conhecimento popular nas indicações de plantas medicinais para tratamento de enfermidades de animais de companhia. Revista Brasileira de Biologia e Farmácia, v. 7, n. 2, p. 66-73, 2012.

VASCONCELOS, J.; VIEIRA, J.G.P.; VIEIRA, E.P.P. Plantas Tóxicas: Conhecer para Prevenir. Revista Científica da UFPA, v. 7, n. 1, 2009.

VEIGA JÚNIRO, V.F.; PINTO, A.C.; MACIEL, M.A.M. Plantas medicinais: cura segura? Química Nova, v. 28, n. 3, p. 519-528, 2005.

VENDRUSCOLO, G.S.; MENTZ, L.A. Levantamento etnobotânico das plantas utilizadas como medicinais por moradores do bairro Ponta Grossa, Porto Alegre, Rio Grande do Sul, Brasil. IHERINGIA, Série Botânica, v. 61, n. 1-2, p. 83-103, 2006.

VERDAM, M.C.S.; SILVA, C.B. O estudo de plantas medicinais e a correta identificação botânica. Visão Acadêmica, v. 11, p. 7, 2010.

ZAGO, J.A.A.; USHIMARU, P.I.; BARBOSA, L.N.; JÚNIOR, A.F. Sinergismo entre óleos essenciais e drogas antimicrobianas sobre linhagens de Staphylococcus aureus e Escherichia coli isoladas de casos clínicos humanos. Revista Brasileira de Farmacognosia, v. 19, n. 4, p. 828-833, 2009. 